\title{
Optimization of biolistic bombardment parameters for Dendrobium sonia 17 calluses using GFP and GUS as the reporter system
}

\begin{abstract}
Genetic transformation system of Dendrobium Sonia 17 was optimized using green fluorescent protein (GFP) and $\beta$-glucuronidase (GUS) gene as the reporter systems. The $35 \mathrm{~S}$ sgfp-TYG-nos (p35S) and pSMDFR, carrying sgfp and gusA gene, respectively, were cobombarded into the calluses. Parameters optimized were acceleration pressure, target distance, gold particle size, pre-bombardment cultured time, plasmid DNA precipitation, total plasmid DNA and the ratio of the plasmids co-bombarded. Both reporter systems responded similarly to the bombardment parameters investigated. Based on the GUS/GFP spot counts, the GFP expression rate was higher than that for GUS under the control of the same promoter, CaMV $35 \mathrm{~S}$. GFP could be used as the reporter system for the co-bombardment as it was rapid and non-destructive system to monitor the transformed tissues. A combination of GFP and antibiotic resistance gene was used to select stable putative transformants. (C) Springer 2005.
\end{abstract}

Keyword: Bombardment; Dendrobium; Green fluorescent protein; Hygromycin 